\title{
Assessment of Completeness of Prescription and Rational Drug Use Practice at Felege Hiwot Referral Hospital, North West Ethiopia
}

\author{
Biset Asrade \\ Affiliation: Department of Pharmacy, College of Medicine and Health Sciences, \\ Bahir Dar University, Bahir Dar, Ethiopia
}

\begin{abstract}
Background: Rational drug use is a tool through which safe, effective and economic medication is provided. Rational prescribing and dispensing ensure adherence to treatment and protects drug consumers from unnecessary adverse drug reactions.

Objective: The assessment of the rational drug use practice and completeness of prescription is important to identify the most common drug use problem and propose appropriate interventions. Methodology: A combination of retrospective and prospective quantitative cross sectional study was conducted in outpatient pharmacy .Drug containing prescriptions ( $\mathbf{n = 6 6 9 )}$ prescribed from July 1, 2014 to September 30, 2015 were selected by systematic sampling technique for prescriber indicators analysis and patients interviewed $(\mathbf{n}=\mathbf{9 0})$ for patient care indicator analysis.

Results: The mean number of drugs per prescription was 1.46; percentage of prescriptions containing less than two drugs per prescription was $85.05 \%$. Antibiotics and injection were prescribed in prescription were $42.04 \%$, $0.73 \%$ respectively. The weight of the patient was omitted $100 \%$ and the diagnoses of $87 \%$ were not written. The strength and duration of the drug were clearly stated in $81.89 \%, 74.61 \%$ of the prescriptions respectively. $32.74 \%$ and $20.93 \%$ of the prescriptions contain the name of the prescribers and dispensers respectively. $80(88.89 \%)$ of patients interviewed had adequate knowledge of how to take the medication prescribed. From all drugs received by the patients only $68.03 \%$ were adequately labeled. The average counseling and dispensing time was 1 min $20 \mathrm{sec}$ and $11 \mathrm{~min} 54 \mathrm{sec}$ respectively.

Conclusion: From the results of the study, we can be concluded that not all prescriptions were complete as few of them lack the necessary information. So there is a need for managerial and educational intervention to improve rational drug use and to enhance good prescribing and dispensing practices.
\end{abstract}

Keywords: rational drug use, patient care indicators, prescribing indicators, EDL, tracer drug

DOI: $10.7176 / \mathrm{JHMN} / 60-02$

Publication date:March $31^{\text {st }} 2019$

\section{Introduction}

The rational use of drugs requires that "patients receive medications appropriate to their clinical needs, in doses that meet their own individual requirements for an adequate period of time, at the lowest cost to them and their community" (1). The irrational use of drugs is a problem, and to manage it the World Health Organization (WHO) convened an international conference in Nairobi, Kenya, in1985 to develop useful guidelines $(1,2)$

Medicines play an important role in health care delivery and disease prevention. The availability and affordability of good quality drugs along with their rational use is needed for effective health care. However, irrational drug use is prevalent, especially in the developing countries due to irrational prescribing, dispensing, and administration of medications (1).

The indicators of prescribing practices measure the performance of healthcare providers in several key dimensions related to the appropriate use of drugs (1). Rational drug use is a tool through which safe, effective and economic medication is provided. It is promoted by the collaborated efforts of prescribers, dispensers and drug consumers.

Rational prescribing ensures adherence to treatment and protects drug consumers from unnecessary drug adverse reactions. Rational dispensing on the other hand, promotes the safe, effective and economic use of drugs (2).

The inappropriate drug use is characterized by the use of drugs when no therapy is indicated, the use of the wrong drug for a specific condition requiring drug therapy, the use of drugs with doubtful/ unproved efficacy, the use of drugs of uncertain safety status, failure to provide availability of safe and effective drugs, the use of too many Medicines per patient(poly- pharmacy), Inappropriate use of antibiotics, often inadequate dosage, over dosage for nonbacterial infections, the use of correct drugs with incorrect administration, dosages and duration, Over use of injections when oral formulations would be more appropriate and Failure to prescribe in accordance with clinical Guidelines $(3,4,5)$. Irrational use of medicines can stimulate inappropriate patient demand, and lead to reduced access and attendance rates due to medicine stock outs and loss of patient confidence in the health level 
$(6,7)$.

Patient care indicators such as average consultation time, average dispensing time, percentage of patients' knowledge of correct dosage w/c understand the way drugs are used and it is important to consider what takes place at health facilities from both the provider's and the patient's perspective. The patient care indicator address key aspects of what patients experience at health facilities, and how well they have been prepared to deal with the pharmaceuticals that have been prescribed and dispensed. The time that prescribers and dispensers spend with each patient sets important limits on the potential quality of diagnosis and treatment. Patients for whom pharmaceuticals are prescribed should, at a minimum, receive well-labeled medications, and should understand how to take each drug (11).

\section{Methods \\ Study design and study Area}

A combination of retrospective and prospective quantitative cross sectional studies was applied in the hospital by selected sample prescription from outpatient pharmacy of the hospital over a three month period from July 1, 2014 to September 30, 2015 to assess rational drug use and completeness of prescription as well as the prescribing and dispensing practices at the hospital. In a retrospective review, this method is done by examining the prescriptions and medicine envelopes and prospective method is done by interview the patients who came to Outpatient pharmacy to review patient care indicators. Data for computation of core prescribing and dispensing were collected as per the recommendations of WHO guide line for investigating drug use at the health facilities (21).The study was conducted at Felege Hiwot Referral Hospital which is located in the center of Amhara region.

\section{Data Collection Tool and Process}

Data was collected by a four Pharmacy personnel who had been adequately trained on how to collect data and supervised throughout their activity by supervisor. They collected the data using WHO standard data collection formats of prescribing indicators, patient care indicators and facility indicator. The data on prescribing indicators were collected retrospectively using prescriptions, and for facility indicator observing the availability of standard treatment guidelines and Formularies in the Hospitals and taking the Hospitals yearly data on availability of Tracer drugs and for patient care indicators by observing and asking closed ended questions directly the patients with their dispensed drugs prospectively.

\section{Data Analysis}

All data in the ordinary prescribing and patient care indicator recording form was entered in to Microsoft Excel 2007, data collection formats and summarized using standard summary forms. All the required statistical analysis was carried out with SPSS version 16.0.

\section{Ethical-Clearance}

Ethical clearance was obtained from drug and therapeutic committee of the Hospital. It is a service improvement and quality assurance work for the benefit of the patient. In addition permission was formally obtained from the medical director's office through a letter of collaboration from department of Pharmacy, Felege Hiwot Referral Hospital. Ethical clearance: Informed verbal consent was obtained. Moreover no participant of the study were mentioned or identified by name or other identifier.

\section{Results}

\section{Completeness of Prescription}

\subsection{Patient related information}

Out of the 669 prescriptions $667(99.70 \%)$ had the name of the patient, and $87(13.00 \%)$ of prescription have diagnosis of the patient, But none of the prescriptions have Address and weight of the patient. 
Table1: Summary of Prescription Paper Issued With Patient Related Information at Felege Hiwot Referral Hospital October 1, 2015 to October 30, 2015. $(\mathrm{N}=669)$

Standard prescription paper used $=669$

Patient information

\begin{tabular}{|l|l|l|l|}
\hline Indicator studied & Number(n) & Percentage (\%) & Gold standard \\
\hline Name of patient & 667 & $99.70 \%$ & $100 \%$ \\
\hline Age & 619 & $92.53 \%$ & $100 \%$ \\
\hline Sex & 616 & $92.08 \%$ & $100 \%$ \\
\hline Address & 0 & $0 \%$ & $100 \%$ \\
\hline Diagnosis & 87 & $13.00 \%$ & $100 \%$ \\
\hline Card number & 452 & $67.56 \%$ & $100 \%$ \\
\hline Date & 495 & $73.99 \%$ & $100 \%$ \\
\hline Weight & 0 & $0 \%$ & $100 \%$ \\
\hline
\end{tabular}

\subsection{Drug related information}

Out of 1099 drugs issued in 669 prescriptions, the correct name, and strength of the drug were clearly stated in $800(72.79 \%)$, and $900(81.89 \%)$ of the prescriptions respectively. But the quantity of the drug was mentioned was very low $36.94 \%$.

Table 2: Summary Of Prescription Paper Issued With Drug Related Information Felege Hiwot Referral Hospital October 1, 2015 To October 30, 2015. $(\mathrm{N}=669)$

No of drugs per prescriptions $=1099$

\begin{tabular}{|l|l|l|l|}
\hline Parameters & Number (n) & Percentage (\%) & Gold standard \\
\hline Correct name & 800 & $72.79 \%$ & $100 \%$ \\
\hline Strength & 900 & $81.89 \%$ & $100 \%$ \\
\hline Dose & 500 & $45.49 \%$ & $100 \%$ \\
\hline Frequency & 870 & $79.16 \%$ & $100 \%$ \\
\hline Duration & 820 & $74.61 \%$ & $100 \%$ \\
\hline Quantity & 406 & $36.94 \%$ & $100 \%$ \\
\hline
\end{tabular}

\subsection{Prescriber and dispenser related information}

In this study, from 669 prescription paper $219(32.74 \%), 140(20.93 \%)$ of the prescriptions contain the name of the prescriber and dispenser in their respective order. As shown from the results below.

Table 3: Summary of Prescription Paper Issued With Prescriber And Dispenser Related Information at Felege Hiwot Referral Hospital October 1, 2015 to October 30, 2015. (N=669)

\begin{tabular}{|l|l|l|l|l|l|}
\hline Prescriber information & \multicolumn{3}{|l|}{ Dispenser information } & \\
\hline Parameters & Number $(\mathrm{n})$ & Percentage (\%) & Number(n) & Percentage & Gold standard \\
\hline Name & 219 & $32.74 \%$ & 140 & $20.93 \%$ & $100 \%$ \\
\hline Signature & 454 & $67.86 \%$ & 247 & $36.92 \%$ & $100 \%$ \\
\hline
\end{tabular}

\section{2 .Prescribing Indicators}

Out of 1099 drugs issued in 669 prescriptions, $0.73 \%$ of the prescription contains injection and the average number of drugs prescribed per prescription (encounter) was 1.64 as shown the table below.

Table 4: Summary Of The Prescribing Practices at Felege Hiwot Referral Hospital, October 1,2015 to October 30,2015. $(\mathrm{N}=669)$

Total number of prescription analyzed $=669$

No of drugs per prescriptions $=1099$

\begin{tabular}{|l|l|l|l|}
\hline Parameters & Number(n) & Percentage (\%) & Gold standard \\
\hline Average No of drugs per Prescription & 1099 & 1.64 & $<2$ \\
\hline Number of antibiotics & 462 & $42.04 \%$ & $<25 \%$ \\
\hline Number of injection & 8 & $0.73 \%$ & $<13$ \\
\hline Number of generic & 1003 & $91.26 \%$ & $100 \%$ \\
\hline Number of EDL & 1099 & $100 \%$ & $100 \%$ \\
\hline
\end{tabular}

\section{Patient Care Indicators}

The study population constituted 90 patients, out of which $31(34.44 \%)$ are female patients and 59(65.56\%) are male patients .About $34(37.78 \%)$ of patients were illiterate as shown in table6.

From 90 interviewed patients, $80(88.89 \%)$ of patients were able to repeat the correct dosage schedule of the 
drugs they had receive and 83(92.22\%) were satisfied by outpatient Pharmacy service of the hospital.

Table 6: Summary on the Result of Patient Care Indicators, Felege Hiwot Referral Hospital, October 1, 2015 to Octoer 30, 2015. ( $N=90)$

\begin{tabular}{|l|l|l|l|}
\hline Indicator studied & Number & Percentage & WHO standard \\
\hline Interviewed patients $=90$ & 147 & $100 \%$ & $100 \%$ \\
\hline No .of drugs prescribed & 145 & $98.64 \%$ & $100 \%$ \\
\hline No. of drugs actually dispensed & 100 & $68.03 \%$ & $100 \%$ \\
\hline No .of drugs adequately labeled & & $37.78 \%$ & \\
\hline Educational status of interviewed patients $=90$ & 34 & $17.78 \%$ & \\
\hline No of patients not able to read and write & 16 & $44.44 \%$ & \\
\hline <less than high school(<grade 9) study & 40 & \\
\hline >high school ( $>$ grade 9) study &
\end{tabular}

\begin{tabular}{|l|l|l|l|}
\hline \multicolumn{4}{|l|}{} \\
\hline Patient having knowledge on the dispensing drugs=90 & number & Percentage & WHO standard \\
\hline Indicator studied & 15 & $16.67 \%$ & $100 \%$ \\
\hline Name of the drug & 86 & $95.56 \%$ & $100 \%$ \\
\hline Frequency & 63 & $70 \%$ & $100 \%$ \\
\hline Duration & 52 & $57.78 \%$ & $100 \%$ \\
\hline Indication & 22 & $24.44 \%$ & $100 \%$ \\
\hline Precaution & 78 & $86.67 \%$ & $100 \%$ \\
\hline Dose & \multicolumn{3}{|l|}{} \\
\hline Patient satisfaction $=90$ & 83 & $92.22 \%$ & \\
\hline Satisfied & 7 & $7.78 \%$ & \\
\hline Not satisfied & 7 & \\
\hline
\end{tabular}

\section{Health Facility Indicators}

\section{4a. Availability of EDL/Formulary and STG}

The purpose is to indicate the extent to which copies of the national essential drugs list or local formulary are available at health facility. These materials were available in the facility (hospital).

4b. Availability of Key Drugs/tracer drugs

From fifty essential drugs, $90 \%$ of the key drugs were available and also from thirteen types of national tracer drugs, $92.31 \%$ of the national tracer drugs were available in the hospital as shown below.

Table 7a: Summary of Health Facility Indicators, at Felege Hiwot Referral Hospital, October 1-30, 2015.

\begin{tabular}{|l|l|l|}
\hline Items & Availability & WHO standard \\
\hline Essential drug list & Yes & Yes \\
\hline Formulary 2013 & Yes & Yes \\
\hline Standard treatment guide line 2014 & Yes & Yes \\
\hline TB treatment guide line & Yes & Yes \\
\hline HIV/AIDS treatment guide line & Yes & Yes \\
\hline National Tracer Drugs & Yes & \\
\hline Amoxicillin tablets & Yes & Yes \\
\hline ORS & Yes & Yes \\
\hline Artemether/lumefantrine tablets & Yes & Yes \\
\hline Paracetamol tablets & Yes & Yes \\
\hline Mebendazole tablets & Yes & Yes \\
\hline TTC eye ointment & Yes & Yes \\
\hline Refampicin+isonizide+pyrazinamide+Ethambutol & Yes & Yes \\
\hline Medroxy progesterone (depo) injection & Yes & Yes \\
\hline Ergometrine injection & Yes & Yes \\
\hline Ferrous gluconate tablets & Yes & Yes \\
\hline Penta valant DPT-hep-Hib vaccine & No & Yes \\
\hline Zinc sulphate tablets & Yes & Yes \\
\hline Gentamycin injection & were & \\
\hline
\end{tabular}

From ten types of the hospital tracer drugs, $90 \%$ of them were available in the hospital as shown below in the table. 
Table 7b: Summary of Health Facility Indicators, Felege Hiwot Referral Hospital, October 1-30, 2015

\begin{tabular}{|c|c|c|}
\hline \multicolumn{3}{|c|}{ Hospital tracer drug availability } \\
\hline Items & Availability & WHO standard \\
\hline TAT1500IU & No & Yes \\
\hline Ceftriaxone 1 gram injection & Yes & Yes \\
\hline Lent-Insuline 1000IU & Yes & Yes \\
\hline Normal saline $1000 \mathrm{IU}$ & Yes & Yes \\
\hline $40 \%$ dextrose & Yes & Yes \\
\hline Cimetidine $200 \mathrm{mg}$ injection & Yes & Yes \\
\hline Lidocine $2 \%$ in $20 \mathrm{ml}$ injection & Yes & Yes \\
\hline Ergometrine injection & Yes & Yes \\
\hline Oxytocin injection & Yes & Yes \\
\hline Atropine sulphate injection & Yes & Yes \\
\hline
\end{tabular}

\section{Discussion}

\section{Completeness of Prescription}

A prescription is an important therapeutic transaction between the prescriber and drug consumer through dispenser. Complete prescription should include name, level of health institution and the main information: patient related information (name, card number, address, sex, age and diagnosis), drug related information (name, strength, dosage form, frequency and duration of treatment), and prescriber and dispenser name, and signature (22).

1a. Patient related information: It refers to those which are included on the prescription and identify patients. In our finding, 99.70\% of patient name was written, it is better than in Saudi Arabia $96.60 \%$ but less than GUH 99.83\% and Debremarkos Referral Hospital $100 \%$ (17, 20, and 21). It also low to the WHO standard which is $100 \%$. WHO standard states that all prescription must contain the full name of the patients.

The diagnosis is important for pharmacists to know the consistency of diagnosis and drugs and to know potential drugs interaction and contraindication (23). However, diagnosis was written only in $87(13.00 \%)$ of the cases $\mathrm{w} / \mathrm{c}$ is very low to the WHO standard and Debremarkos referral Hospital $98.70 \%$ but very higher than GUH $0.01 \%$. Including diagnosis on the prescription will help the pharmacist to contribute to positive therapeutic outcomes through avoiding in- advertent prescribing and medication errors. Making the write diagnosis is the corner stone for choosing the right kind type of therapy (22).

It is apparent that rational selections of drugs' dose and dosage forms would be highly determined by the age of the patient. The dose that should be administered to children would naturally be different from those given to adults, since age plays an important role in a successful management of the therapy (24). If the pharmacists dispenses the drug without asking the age of the patient, he/she might wrongly dispense an adult dose to a child and vice versa, hence causing in either a therapeutic failure or over dosage causing toxicity. The result is an indication that some prescribers need to be reminded of the scientific rational behind dose optimization versus patient age. As shown in table 1 above, age and card number were written in 619(92.53\%) and $452(67.56 \%)$ of all prescriptions respectively but it is higher than in Saudi Arabia age,77.30\% and GUH age, $67.93 \%$ but lower than Debremarkos referral hospital age, $98.1 \%$ and also the card number lower than GUH $73.54 \%(15,19,20)$. In our finding both age and card number value is lower than WHO standard. All prescriptions should have contained age and card number according to WHO standard. Our finding shows that there was problem on age dependant drug uses so we will prevent therapeutic failure and toxicity by promoting rational prescribing and dispensing. Sex of the patients should also be specified on all prescriptions as some medicines could have sex dependent pharmacokinetic profiles (25). In our study only616 (92.08\%) of all prescriptions had sex w/c higher than Saudi Arabia 51.30\% and GUH67.93\% but lower than Debremarkos Hospital 97.7\%. Although all prescriptions should have contained sex according to WHO standard so thus data needs to be improved as part of promoting the rational drug prescribing.

Weight of the patients should be specified on all prescription all most all medicines could have both pharmacokinetics and pharmacodynamics weight dependant profiles. Although the concept of varying the dose with the body weight has a long tradition, adult doses have been assumed to be the same irrespective of the size or shape. Yet adult weights vary two to three fold, while a large fat mass can store large excessive of highly lipid soluble drugs compared to lean patients of the same weight. Since weight plays an important role like age in a successful management of the therapy (24). If the pharmacists dispenses the drug without asking the weight of the children, he/she might wrongly dispense an adult dose to a child and vice versa, hence causing in either a therapeutic failure or over dosage causing toxicity. The result is an indication that some prescribers need to be reminded of the scientific rational behind dose optimization versus patient weight. In FHRH, none of the prescription contains the weight of the patient but in Debremarkos referral Hospital1.04\%\% had weight of the patients. But according to WHO standard all prescription should have been contain the weight of the patients b/c 
all patients medication dose calculated based on weight especially for children's. So thus data needs to be improved as part of promoting the rational drug prescribing and the right dose of dispensing to prevent therapeutic failure and toxicity.

1b. Drug related Information: The prescription was checked for having complete information on the name, dose, frequency and duration of prescribed drugs together with legibility. Ideally, all information on the prescription should be fulfilled clearly, legibly without any ambiguity (22). In our finding. As shown in the above table (2), out of 1099 drugs issued in

669 prescriptions, the correct name and strength of the drug were clearly stated in $800(72.79 \%)$ and $900(81.89 \%)$ of the prescriptions respectively w/c is lower than in Saudi Arabia correct name , $100 \%$ and in Debremarkos Hospital correct name and strength $100 \%$ and $84 \%$ respectively but the strength is higher than Saudi Arabi47.20\% . In our finding $27.21 \%$ of them were not clearly stated. Mostly the prescriber write the drug name by abbreviation. But, the dose, frequency and durations of the drug were clearly indicated in $45.49 \%, 79.16 \%$ and $74.61 \%$ of the prescriptions respectively. In Saudi Arabia dose 19.4\%, in Debremarkos Hospital dose, frequency and duration was 93\%, 92.2\% and 90\% respectively w/c is higher than Saudi Arabia but lower than Debremarkos Hospital findings. But the quantity of the drug was mentioned in our finding was very low $36.94 \%$ to Debremarkos Hospital 98\% $(15,19)$. When we compare it with WHO standard, all drug related information should have been written on the prescription paper but not in our finding. This indicates that the trend on the improvement of the issuance of the prescription with complete and clear information was not satisfactory.

1c. Prescriber Information: A prescriber is not always a medical doctor; he/she can be a paramedical worker such as a medical assistant, a midwife, or a nurse who makes a diagnosis and orders drug(s) to patients. Therefore, in order to identify the person involved in prescribing, the prescription should include the prescriber's name, qualification, signature and the date on which the prescription is written. Prescriber's name and address indicated on the prescription will allow either the patient or the dispenser to contact the prescriber for any clarification or potential problem with the prescription (22). Regarding to the prescriber information; the name of the prescriber was not well indicated on some of the prescriptions posing the issue of accountability in the medico legal system as shown from the result above table (3). This information will be important for cross check evaluations in cases of prescriptions errors and help in easy identification of the liable prescriber. In our finding, 219(32.74\%), $454(67.86 \%)$ of the prescriptions contain the name, signature, of the prescriber in their respective order $\mathrm{w} / \mathrm{c}$ is lower than GUH name of prescriber and signature $33.42 \%$ and $96.69 \%$ respectively(20). Even if they wrote their name and signature, it was difficult to read the prescriber name and identify their signature $\mathrm{b} / \mathrm{c}$ the name and signature of prescriber were not written properly. Based on WHO standard, all prescriber should have written their name and put their signature in order to identify potential problem with the prescription and any clarification by contacting either the patients or dispensers.

1d .Dispenser Information: Likewise, the dispenser is not always a pharmacist but also can be a pharmacy technician and an assistant who is licensed to or authorized to dispense drugs. It is the primary responsibility of the pharmacist to assure the correct dispensing of the drug and maintaining the quality of the drug dispensed. Dispensing error may be common when the dispensing is performed at low level of health care provider. Therefore, information which used to identify the dispenser who issued the drug to the patient (including the dispenser's name and signature) on the prescription should be briefly recorded. From the 669 prescriptions, only

$140(20.93 \%)$ prescriptions contain dispensers' name and $247(36.92 \%) \mathrm{w} / \mathrm{c}$ contain dispensers' signature- table (3). It is very low to WHO standard so, this assessment indicates that dispenser information was very poor in this hospital but better than Gondar University Hospital reviewed dispensers' name and signature $0 \%$ and $0.35 \%$ respectively (20). So all dispensers should have written their name and signature to respond any drug related problems.

\section{Prescribing Indicators}

The three core drug use indicators of WHO were used to assess the drug use problem in hospital.

2a. Average number of Drugs per Prescription: Prescribing multiple drugs to patients at once (technically called poly-pharmacy) it may lead missing of dose, over dosing and drug-drug interaction or drug food interaction. The average number of drugs prescribed per prescription (encounter) was 1.64 (minimum 1 and maximum 5) and \% of prescription contain $\leq 2$ drugs per prescription $85.05 \%$. The average number of drugs per encounter is better than In Nepal, ChinaIndia, Debremarkos Hospital and GUH 2.5, 3.5, 2.9, 1.9 and 1.76 respectively $(14,15,16,19$, 20 ) and also better than in Hawassa hospital number of drugs per encounter was 1.9 (maximum number of drugs per prescription 4 and minimum number of drugs per prescription was 1). The percentage of prescription contain $\leq 2$ drugs per prescription is better than Gondar University Hospital (maximum number of drug per prescription 7 , minimum number of drug per prescription

1 , $\%$ of prescription contain $\leq 2$ drugs per prescription $80.87 \%$ ). This result shows presence of acceptable prescribing practice based on WHO recommendation of less than two drugs per prescription.

2b. Percentage of Drugs prescribed by Generic Name: in Felege Hiwot Referral Hospital 1003 (91.26\%) of 
drugs were prescribed by generic name w/c is better than in western Nepal, the generic name percent was $13 \%$ (14), while in India, the percentage was 73.4\%(16) and in Saudi Arabia 19.9\%(15) but lower than in China 96.12\%(15), in Hawassa University Teaching and Referral Hospital 98.7 \%, in Debremarkos Hospital 93\% and in Gondar University Teaching and Referral Hospital 99.16\%(18, 19, 20). However, the indicator could reach 94\% (25).Even if it is less than from Ethiopian Hospitals reviewed above; it was found to be very encouraging but it needs further intervention to achieve the WHO standard.

2c .Percentage of Prescriptions with an Antibiotic Prescribed: As shown above table (4), the antibiotic \% in the Felege Hiwot Referral Hospital was 462 (42.04\%). The antibiotics use pattern of FHRH is lower than in some countries and Ethiopian Hospitals like the antibiotic prescriptions \% in China, Nepal, India and Gondar teaching referral Hospital was $29.9 \%, 28.3 \%, 39.6 \%$ and $29.14 \%$ respectively. But it is better than, in Hawassa University Teaching and Referral Hospital 58.1\% and in Debremarkos Referral Hospital42.2\% (19, 20).Our finding shows that the use of antibiotics was higher than the WHO standard $(<25 \%)$. The overuse of antibiotics is a waste of resource. It also results in significant patient harm in terms of poor patient outcomes and adverse drug reactions. The overuse of antibiotics is leading to increased antibiotics resistance in order to prevent thus problems we should use properly.

2d. Percentage of Prescriptions with an Injection Prescribed: patients who came to the outpatient pharmacy have gotten the service, so this could inflate the use of injection standard from outpatient pharmacy. The injection percent, in Felege Hiwot referral hospital was $8(0.73 \%)$, while the indicator was higher in some countries. However, the indicator ranged from 0.2 to $48 \%$ among different countries (26). According to WHO standard (13.4$24.1 \%)$ when emergency prescription is included if not WHO standard is less than $13 \%(<13 \%)$ for outpatient. The use of injections was so better in this Hospital w/c has value within the WHO standard and also better than other countries and Hospitals like Nepal 31\%, China 20.02\%, Hawassa Hospital 38.1\%, Debremarkos Hospital 10\% and GUH $28.5 \%$ but lower than in India $0.2 \%$. Excessive and unnecessary use of injections is expensive in terms of health care cost to patients, health staff time and sterilization of equipment. Inject able drugs can be complicated by injection abscess, paralysis, and infection with deadly viruses such as AIDS. This injection use at this hospital may be due to the reason that most of the time patients admitted to the hospital were in a serious disease condition requiring an urgent and quick acting medication.

2e. Percentage of Drugs prescribed from Essential Drug List: In our finding, all drugs prescribed from the hospital essential drug list (EDL).WHO recommended that $100 \%$ of drugs should be prescribed from EDL. The percentage of drugs prescribed from EDL in Nepal, China, and India was $32.8 \%, 48.85 \%$ and $90.3 \%$ respectively and also in Ethiopian hospitals like GUH Hospital and Hawassa University Hospital ,The percentage of drugs prescribed from EDL were $98.89 \%$ (20 and 96.6 \% (18) respectively. So, the use of EDL in FHRH is better than other countries and Ethiopian Hospitals reviewed above but the same as Debremarkos referral hospital and it is best trend and encouraging.

\section{Patient Care Indicators}

In our finding, the study population constituted 90 patients, out of which $31(34.44 \%)$ are female patients and $59(65.56 \%)$ are male patients .About 34(37.78\%) of patients were illiterate as shown in table (6).

3a. Average Dispensing Time: The purpose is to measure the time that medical personnel (pharmacist or pharmacy technician) spend with the patients during dispensing drugs in the pharmacy. Time was recorded during the dispensing drugs to the patient, that is, the time between arriving at the dispensary counter and leaving.

Based on WHO standard, the average dispensing time should be $>5$ minutes. But in our finding the average dispensing time was found to be 11.54 minutes. When we compared to other studies like Nepal 52 minute, GUH 4.3 minutes the dispensing time was within the WHO standard and counseling time was 1.20 minutes. Since, there was a recording of patients name, sex, age, area where they come, identification number, types of drugs prescribed with internal ID, units and strengths, retail price and total price from cash sales ticket this made the time to be somewhat longer.

3b. Percentage of Drugs adequately labeled: The purpose is to measure the degree to which dispensers' record essential information on the drug packages they dispense. A label should be considered adequate if it confirms all the requirements (name, strength, dose, duration and quantity of the drug dispensed). The main function of a label on a dispensed drug is to uniquely identify the contents of the container and to ensure that the patient have clear and concise information about the use of the drug. Each dispensed drug must be appropriately labeled to comply with legal and professional requirements. According to our finding, the hospital average percentage of drugs adequately labeled were $100(68.03 \%) \mathrm{w}$ /c higher than In Nepal, $1.4 \%$ in India $18.5 \%$,in Gondar University Hospital $8.47 \%$. These results have shown there is somewhat good practice in this hospital than other countries and Ethiopian Hospitals reviewed above but it is lower than the WHO standard. When we see our finding, mostly dispensers label on the paper bag but not strip and bottles w/c contains syrups. So there is a legal requirement to be added on the label of any prescribed or over the counter medicines: Generic name, total quantity, direction for use, route of administration, precaution relating to the use, and storage of the medicine. If the product is dispensed 
more than one, it is better to differentiate each other and also in our finding, 34(37.78\%) of clients were illiterate, dispensing drugs without label, incomplete label, illegible label will obviously increase the chance of medication error, drug related adverse events and therapeutic failure.

3c. Patient knowledge of Correct Dosage: The purpose of the indicator is to measure the effectiveness of the information given to patients on the dosage schedule of the drugs they receive. In our finding, 86(95.56\%) of patients were able to repeat the correct dosage schedule of the drugs they had received and better than studies conducted in other countries and hospitals (in Nepal 81\%(14) and India 80.5\%)(16) and in GUH 80\%(20) of patients interviewed had adequate knowledge of how to take the medication prescribed but lower than WHO standard and also in our finding, $16.67 \%$ of the patient recall the name of the medication w/c lower than in Botswana 31\%(17), in Debremarkos referral Hospital 17\% (19)and in Gondar University Hospital 19.35\%(20).

In our finding, 95.5\% recall the frequency of the drug w/c lower than in Debremarks referral hospital $99 \%$. $70 \%$ know the duration of the treatment w/c higher than in Botswana $44 \%$ but lower than in Debermarkos referral hospital $93 \% .57 .78 \%$ of them they know the use of the medication (indication) w/c lower than in Botswana $92 \%$. Among of 90 patients $24.44 \%$ know the precaution w/c lower than in GUH $61.29 \% .86 .67 \%$ also know the dose of the medication w/c lower than in Debremarkos referral hospital 95\% but better than in Botswana $83 \%$. Due to inadequate labeling of drugs during dispensing and counseling, no confirming their drug instructions at the time of dispensing and also low educational status of the patients contributes to low adherence to treatments and inadequate knowledge about their medication.

3d. Percentage of drugs actually dispensed: In our finding, from the total of $147 \mathrm{drugs}$ were prescribed, $145(98.64 \%)$ were dispensed $w / c$ is higher than in India $76.9 \%$ and GUH 52(89.66\%).All most all drug available in the Hospital.

3e. percentage of patient satisfaction: In our finding, From 90 interviewed patients $83(92.22 \%)$ were satisfied and $7(7.78 \%)$ were dissatisfied but in Debremarkos referral hospital 93\% patients were satisfied with the dispensers' counseling (19). Therefore we will do more to satisfy almost all patients.

\section{4 .Health Facility Indicators}

The ability to prescribe drugs rationally is influenced by many features of the working environment. An educate supply of essential drugs and access to unbiased information about these drugs are particularly important. Without these it is difficult for health personnel to function effectively.

4a .Availability of EDL/Formulary and some treatment guide line

The purpose is to indicate the extent to which copies of the national essential drugs list or local formulary or HIV/AIDS treatment guide line and TB treatment guide line were available at health facility. These materials were available in the facility $\mathrm{w} / \mathrm{c}$ used to promote rational drug use.

\section{4b .Availability of Key/tracer Drugs}

The purpose was to measure the availability at health facilities of key drugs recommended for the treatment of some common health problems. Access to essential drugs is one of the basic requirements for delivery of proper health care. To measure the physical availability of essential drugs, 50 essential drugs which were used to treat the most common health problems in the catchment area were selected. So, $90 \%$ of key drugs were available which better than GUH 68 essential drugs, $66 \%$ of key drugs were available

Access to essential /tracer drugs is one of the basic requirements for delivery of proper health care. So all national and hospital tracer drugs were available in the facility (hospital) except zinc sulphate tablets and TAT respectively as shown table7a $\boldsymbol{\&} \mathbf{7 b}$.

Availability of drug is also one of the main factors that influence prescribing practice. Therefore establishing a system to maintain continuous supply of drugs included on the formulary list is useful to avoid prescribing alternative medicines with limited comparative studies.

\section{Conclusion}

From the results of the study, it can be concluded that not all prescriptions were complete as few of them lack the necessary patient related information (sex, age, weight, patient diagnosis) and drug related information(correct name, strength, dose, frequency, duration, quantity of the drug) and also the name and signature of the prescriber and dispenser.

With regard to the WHO limit of generic and antibiotic use at Felege Hiwot Referral Hospital fails to maintain the limit, so it needs some improvement on generic and antibiotics use pattern in the hospital.

More over the average number of drugs per prescription and number of different types of drugs that were prescribed from Hospital drug list was within the range of the WHO standard therefore it is a best trend and encouraging.

All most all drug available in the Hospital but labeling on each and every dispensed drug to patients and also both good dispensing and counseling practice are low. Therefore, good labeling, dispensing and counseling practice are required to upgrade patient knowledge and adherence to treatment. There was continuous supply of 
tracer drugs including the formulary list and standard treatment guideline w/c useful to avoid prescribing alternative medicines with limited comparative studies.

\section{Acknowledgement}

This review of rational drug use and completeness of prescription at Felege Hiwot Referral Hospital was realized by the collaborative efforts of experts from USAID/SIAPS Ethiopia and pharmacists of Felege Hiwot Referral Hospital.

In this regard, we would like to express our deepest gratitude to the partners such as USAID/SIAPS and those experts involved directly or indirectly in the study of rational drug use and completeness of prescription evaluation.

\section{References}

1. WHO: The Rational Use of Drugs. Report of a conference of experts, Nairobi, 25 - 29 November 1985 .Geneva: World Health Organization; 1987.

2. Food, Medicine and Healthcare Administration and Control Authority (FMHACA) of Ethiopia. 2nd edition. Addis Ababa: Manual for Medicines Good Prescribing Practice; 2012.

3. WHO: WHO Medicines Strategy: Framework for action Essential drugs and Medicines policy $2000-$ 2003 .Geneva: WHO; 2000. (WHO/EDM/2000.1).

4. WHO: The use of Essential Drugs.6th Report of the Expert Committee. Geneva: WHOTechnicalReportSeries850.3; 1995.

5. Hogerzeil HV, Walker GJA, Sallami AO, Fernando G: Impact of an essential drugs program on availability and rational use of drugs.

6. Chukwuani, CM. Onifade, M., Sumonu, K. Survey of drugs use practice and antibiotics prescribing pattern at a general hospital in Nigeria. Pharm world Sci 2002; 24 (5): 188-195

7. The Drug Administration and Control Authority (DACA) of Ethiopia. Zonal Hospital Standard Treatment Guideline 2010. xixii 24

8. AbdulahiM, ShiferawT: Pattern of prescription in Jimma Hospital. Ethiop J Health Dev 1997, 11(3):263-267.

9. Hogerzeil HV, Bimo , Ross-Degnan D, Laing RO, Ofori-Adjei D, Santoso B, Azad ChowdhuryAK, DasAM, Kafle KK, MabadejeAF: Field tests for rational drug use in twelvedevelopingcountries.Lancet1993,342(8884):14081410.

10. WHO: How to investigate drug use in health facilities: selected drug use indicators. Geneva: WHO/DAP/93.1;1993.

11. Laing RO, Hogerzeil HV: Ten recommendations to improve use of medicines in developing countries. Health Policy Plan 2001, 16(1):13-20.

12. WHO: World Health Organization promoting rational use of medicines: core components. WHO Policy and Perspectives on medicine no. 5 Document WHO/EDM/2002.3 .Geneva: WHO; 2002.

13. Debalto D, Gobe Z, Tekle mariam S: A base line survey of prescribing indicators and factors influencing prescribing in southern Ethipia.J Health Dev1991, 11(3):263-267.

14. .Bimo: Field testing of drug use indicators of INRUD: report of field trip to Indonesia, Bangladesh, and Nepal, June-July 1991. In How to investigate drug use in health Facilities .WHO:Geneva;1993.74(WHO/DAP/93.1)

15. Heng Wang, , , Niannian Li, , , etal: Prescription Pattern and Its Influencing Factors in Chinese County Hospitals: A Retrospective Cross-Sectional Study DOI: 10.1371/ journal.pone.0063225.

16..Suni, K.,Punam,S.,Madhuri K.Pattern of prescription and drug dispensing in Lokmanya Medical College and General Hospital,Mumbai,India.Indian Journal of Medicine2005:vol72,No2:117-121.

17. Decosta, A., Bhartiya, S., Nandeswar, S., Diwan, VK. Patterns of drug in the public sector primary health centers of Bhopal district. Pharm. World Sci. 2008, 30 (6): 684-693.

18 Anteneh Assefa Desalegn, completeness of prescription and rational drug use, Hawassa, Ethiopia, BMC Health Services Research 2013, 13:170 doi:10.1186/1472-6963-13-170.

20 Admassie E, etal: Assessment of Drug Use Practices and Completeness of Prescriptions in Gondar University Teaching Referral Hospital. Int J Pharm Sci Res. 2013; 4(1); 265-275.

21. WHO. Action Program on Essential drugs, How to investigate drug use in health facilities. World health organization, Geneva 1993: 1-87

22. Drug Administration and Control Authority of Ethiopia. Standard treatment guide lines for general Hospitals: DACA; January 2010, xvi-xvii.

23. Zou, J., Li ,L., Zhang, C., Yan, Y., Gao, F., and Zhang, H. Analysis of outpatient prescription indicators and trends in Chinese Jingzhou Area between September 1 and 10, 2006-2009. African Journal of Pharmacy and Pharmacology 2011, (2), 270275,

24. Biiungi H. The domestication of injections: A Study of Social Relations of Health Care in Bosoga, Estern Uganda, Institute of Anthropology, University of Copenhagen 1994a, 1-28

25. Chukwuani, CM., Onifade, M., Sumonu, K. Survey of drugs use practice and antibiotics prescribing pattern at 
a general hospital in Nigeria. Pharm world Sci 2002; 24 (5): 188-195.

26. WHO. How to investigate drug use in health facilities: selected drug use indicators, Action Program on Essential Drugs 1993, WHO/DAP/93.1. 